\title{
Arterite de Takayasu e Gestação: Relato de Caso
}

\author{
Takayasu's Arteritis and Pregnancy: a Case Report
}

Mônica de Souza Visniewski Ximenes, Sinval Ferreira de Oliveira, Antônio Vieira Machado, Mário Dias Corrêa, Júlio César de Faria Couto

\begin{abstract}
RESUMO
A arterite de Takayasu é caracterizada pela oclusão idiopática da aorta e de seus principais ramos. A doença apresenta uma predileção por mulheres jovens, sendo, dessa forma, ocasionalmente encontrada associada à gestação. Apresentamos o caso de uma gestante portadora de um grau avançado de arterite de Takayasu cuja gravidez foi acompanhada por uma equipe formada por obstetras e cardiologistas e apresentou evolução satisfatória. Ocorreu apenas uma hospitalização por exacerbação dos sintomas na $32^{a}$ semana de gestação, que foram controlados com tratamento clínico. O parto vaginal ocorreu na $37^{a}$ semana, com nascimento de uma criança pesando $2.750 \mathrm{~g}$. A paciente evoluiu sem complicações clínicas.
\end{abstract}

PALAVRAS-CHAVE: Arterite de Takayasu. Complicações da gravidez. Doenças auto-imunes.

\section{Introdução}

A arterite de Takayasu é uma arteriopatia de etiologia desconhecida que acomete a aorta e seus principais ramos. A natureza da doença leva a quatro complicações principais: retinopatia isquêmica, hipertensão arterial secundária, regurgitação aórtica e aneurisma da aorta ou de seus ramos. O prognóstico das pacientes com complicações graves é reservado, ao passo que pacientes sem complicações ou com complicações moderadas apresentam uma melhor evolução ${ }^{1}$. Conseqüentemente, os problemas relacionados à gestação e ao parto são superpostos às complicações cardiovasculares já existentes. Dessa forma, um cardiologista deve ser consultado com o objetivo de avaliar os riscos da gestação e do trabalho de parto ${ }^{2}$.

Desde a sua primeira descrição em 1908, numerosos casos dessa doença têm sido relatados em diferentes partes do mundo ${ }^{1-3}$. Ela apresenta predileção particular por mulheres jovens, sendo, dessa forma, ocasionalmente encontrada associada à gestação $0^{1,4}$.

Clínica Obstétrica da Maternidade Hilda Brandão, Santa Casa de Belo Horizonte

Correspondência:

Júlio César de Faria Couto

Av. Flávio dos Santos, 335/403 - Floresta

31015-150 - Belo Horizonte - MG
O curso clínico de uma gestação e parto em mulher portadora de arterite de Takayasu é relatado aqui. A influência da gestação e do trabalho de parto no curso clínico da doença e os efeitos da mesma na gestação são analisados.

\section{Relato de caso}

Trata-se de mulher de 29 anos, primigesta, portadora de arterite de Takayasu. A suspeita diagnóstica foi feita em 1994 por apresentar como sintomas tonturas, precordialgia e diminuição dos pulsos nos membros superiores, e confirmada por exames complementares que demonstraram uma hipertrofia do ventrículo esquerdo com dilatação aneurismática da porção inicial da carótida interna e aorta abdominal.

Desde então foi iniciado tratamento com prednisona $(20 \mathrm{mg} / \mathrm{dia})$ e a paciente permaneceu assintomática. Iniciou o pré-natal em agosto de 1998 na $14^{a}$ semana de gestação. A dose de prednisona foi reduzida para $10 \mathrm{mg} /$ dia e a paciente foi acompanhada simultaneamente pelas equipes de Cardiologia e Obstetrícia da instituição. Os exames clínico e laboratoriais de rotina do pré-natal encontravam-se normais.

A paciente permaneceu assintomática até a $32^{a}$ semana de gestação, quando apresentou 
precordialgia e hipotensão arterial, sendo hospitalizada. O ecodopplercardiograma realizado durante a internação demonstrou, além das alterações já conhecidas, uma dilatação moderada da aorta ascendente e regurgitação aórtica leve. A paciente foi classificada como grupo III da arterite de Takayasu. O parto normal foi contra-indicado sendo programada uma cesariana. No entanto, a paciente iniciou trabalho de parto espontâneo na $37^{\mathrm{a}}$ semana, sendo admitida na maternidade em período expulsivo. Prestou-se assistência ao parto, com nascimento de uma criança do sexo feminino, pesando 2.750 gramas. Após o parto, embora se encontrasse clinicamente estável, a paciente foi encaminhada ao CTI para observação, onde permaneceu por 24 horas. Após esse período retornou à maternidade, onde permaneceu por mais quatro dias, recebendo alta hospitalar em boas condições juntamente com o recém-nascido.

\section{Discussão}

A arterite de Takayasu é uma doença inflamatória crônica que afeta a aorta e seus principais ramos, determinando fibrose da camada íntima e adventícia, além de degeneração da camada média. O processo proliferativo provoca alterações luminais obstrutivas, levando a uma dilatação aneurismática pós-estenótica como complicação tardia. Acomete com mais freqüência o arco aórtico e seus ramos, principalmente nos locais de emergência destes ${ }^{2}$.

Apesar de sua etiologia permanecer desconhecida, as semelhanças com o lupus eritematoso sistêmico e a boa resposta à corticoterapia sugerem etiologia auto-imune ${ }^{1}$.

A arterite de Takayasu pode ser classificada de acordo com o sítio anatômico da lesão ou a presença de complicações. A segunda classificação é a preferida, uma vez que avalia melhor o prognóstico da paciente. Considera-se que para os grupos I e IIA há uma sobrevida de $100 \%$ em 10 anos e para os grupos IIB e III de $74,2 \%{ }^{1-3}$ (Tabelas 1 e 2 ).

Tabela 1 - Classificação da arterite de Takayasu conforme o sítio anatômico da lesão

\begin{tabular}{ll}
\hline Classificação & \multicolumn{1}{c}{ Sítio anatômico } \\
\hline Tipo I & Lesões limitadas ao arco aórtico e seus ramos \\
Tipo II & $\begin{array}{l}\text { Há envolvimento da aorta torácica descendente, aorta } \\
\text { abdominal e seus ramos }\end{array}$ \\
Tipo III & $\begin{array}{l}\text { Combinação dos tipos I e II } \\
\text { Tipo IV }\end{array}$ \\
& $\begin{array}{l}\text { Corresponde ao tipo I, II ou III associado a } \\
\text { comprometimento da artéria pulmonar }\end{array}$ \\
\hline
\end{tabular}

Tabela 2 - Classificação da arterite de Takayasu conforme a presença de complicações ${ }^{\star}$ Classificação Complicações

\begin{tabular}{ll}
\hline Grupo I & Ausência de complicações \\
Grupo II & Somente uma complicação está presente: \\
& IIA - Presença de complicação leve \\
& IIB - Presença de complicação grave \\
Grupo III & Presença de duas ou mais complicações
\end{tabular}

* As complicações consideradas para efeito de classificação são: hipertensão arterial secundária, insuficiência aórtica, aneurismas e retinopatia.

A doença apresenta um grande número de sinais e sintomas, havendo um longo intervalo entre o início dos sintomas (normalmente na adolescência) e o diagnóstico definitivo (entre 20 e 40 anos) ${ }^{1}$. Os sinais e sintomas dependem da fase da doença (inicial ou tardia), do sítio anatômico da lesão e da presença de complicações. A fase aguda da doença normalmente ocorre na adolescência. Os sintomas mais comuns são anorexia, febre baixa, mal-estar geral e mialgia. A doença normalmente permanece sem diagnóstico nessa fase e os sintomas são atribuídos a uma doença viral.

O diagnóstico é normalmente feito na fase tardia da doença ${ }^{2}$. A maioria das pacientes são assintomáticas. Os sintomas, quando presentes, incluem sincopes, precordialgia, distúrbios auditivos e visuais, dor sobre o trajeto das artérias e claudicação. O diagnóstico se faz pela anamnese e exame fisico, em que podemos encontrar hipertensão arterial, principalmente quando a artéria renal é afetada; diminuição ou ausência de pulsos nos membros superiores; sinais de insuficiência cardiaca; sopro de regurgitação aórtica ou retinopatia ${ }^{2,5}$.

O diagnóstico é, portanto, essencialmente clínico. Os exames complementares são utilizados para avaliar a repercussão da doença sobre o organismo. A avaliação laboratorial é feita pela velocidade de hemossedimentação (VHS) elevada. Outros achados, também inespecíficos, incluem anemia e hipergamaglobulinemia. Os fatores reumatóides e anticorpos antinucleares são negativos. A aortografia é método complementar indispensável, pois demonstra alterações da doença, como oclusão e aneurismas vasculares. O ecocardiograma também é útil para determinar a hipertrofia ventricular e patologia da válvula aórtica ${ }^{1,4}$.

Para o tratamento utilizam-se corticosteróides, usualmente prednisona na dose de 30 a $50 \mathrm{mg}$ por dia. Na gestação essa dose é reduzida para $10 \mathrm{mg} /$ dia e o controle é feito por meio da VHS. As pacientes dos grupos I e IIA se beneficiam com o tratamento clínico. O tratamento cirúrgico, como exérese dos aneurismas, endarterectomia, by-pass de artérias obstruídas ou troca da válvula aórtica, é reservado para pacientes com 
sintomas graves, principalmente do grupo IIB e III $^{6}$.

As portadoras de arterite de Takayasu que desejam engravidar devem ser esclarecidas a respeito dos riscos de complicações, como a exacerbação da hipertensão arterial já existente, insuficiência cardíaca e nascimento de crianças com baixo peso ${ }^{1-3}$.

A gravidez não altera o prognóstico da doença, mas alguns eventos desfavoráveis como agravamento da hipertensão arterial e falência cardíaca podem ocorrer. A fim de se obter um resultado favorável na gestação é necessário um controle rigoroso da pressão arterial com tratamento antihipertensivo desde o início da gravidez. Préeclâmpsia pode ocorrer por alteração do fluxo placentário, como também por aspectos imunológicos da doença de Takayasu e necessita de um adequado manejo obstétrico ${ }^{1,3}$.

A doença de Takayasu, por sua vez, não aumenta a taxa de cesariana, parto pré-termo ou morte neonatal. Nas pacientes com um grau mais avançado da doença (grupos IIB e III), principalmente com comprometimento da aorta abdominal e artéria renal, pode ocorrer insuficiência placentária, levando ao nascimento de crianças com baixo peso. Estudos têm demonstrado diferença significativa entre o peso das crianças de pacientes dos grupos I e IIA ( $3 \mathrm{~kg}$ ) para aquelas dos grupos IIB e III $(2,5 \mathrm{~kg})^{2,6}$ (Tabela 3$)$.

\begin{tabular}{|c|c|c|c|c|c|c|}
\hline $\begin{array}{l}\mathrm{N}^{0} \text { de } \\
\text { casos }\end{array}$ & $\begin{array}{l}\text { Classificação da } \\
\text { arterite de } \\
\text { Takayasu* }\end{array}$ & $\begin{array}{l}\text { Tipo de parto } \\
(\mathrm{n} / \%)\end{array}$ & $\begin{array}{l}\text { IG média do } \\
\text { parto } \\
\text { (semanas) }\end{array}$ & $\begin{array}{l}\text { Peso médio do } \\
\text { RN (gramas) }\end{array}$ & $\begin{array}{c}\text { Complicação materna durante } \\
\text { a gestação }\end{array}$ & Referência \\
\hline \multirow[t]{4}{*}{33} & 1 & VB $(19 / 58)$ & 39 & 3.023 & HAIG, hemorragia cerebral & 2 \\
\hline & $\| A$ & CST $(6 / 18)$ & 39 & 3.023 & HAIG, exacerbação dos sintomas & \\
\hline & $\| \mathrm{B}$ & VB $(4 / 12)$ & 39 & 2.599 & HAIG, exacerbação dos sintomas & \\
\hline & III & CST (4/12) & 39 & 2.599 & HAIG, exacerbação dos sintomas & \\
\hline \multirow[t]{3}{*}{5} & $\| A$ & CST $(2 / 40)$ & 39 & 2.460 & HAIG, exacerbação dos sintomas & 3 \\
\hline & IIB & VB $(1 / 20)$ & 38 & 2.250 & HAIG, exacerbação dos sintomas & \\
\hline & III & CST $(2 / 40)$ & 38 & 2.250 & HAIG, exacerbação dos sintomas & \\
\hline \multirow[t]{2}{*}{15} & I & VB $(12 / 76)$ & 39 & ND & HAIG & 4 \\
\hline & $\| A$ & CST (3/24) & 39 & ND & HAIG & \\
\hline 3 & $\| \mathrm{A}$ & CST $(3 / 100)$ & 37 & 2.930 & Nenhuma & 1 \\
\hline 2 & I & VB $(2 / 100)$ & 38 & 3.140 & HAIG & 5 \\
\hline 1 & I & CST $(1 / 100)$ & 40 & 3.150 & Nenhuma & 7 \\
\hline 1 & III & VB $(1 / 100)$ & 37 & 2.750 & Exacerbação dos sintomas & Este \\
\hline
\end{tabular}

$\mathrm{RN}=$ Recém-nascido, IG = idade gestacional; HAIG = hipertensão arterial induzida pela gestação; VB = parto via baixa; CST = cesárea segmentar transperitoneal, ND = não definido ${ }^{*}$ Classificação baseada na presença de complicações

A decisão quanto à via de parto deve ser planejada e tanto fatores obstétricos quanto não obstétricos (como a classificação da doença) devem ser levados em consideração. Durante o parto, é necessária a monitorização contínua da pressão arterial devido à ampla flutuação dos niveis pressóricos observada no segundo estágio do trabalho de parto, a qual pode ser controlada com a analgesia peridural, considerada a ideal para essas pacientes ${ }^{1,4,6}$.

O fórceps de alívio também está indicado para abreviar o período expulsivo. A antibioticoprofilaxia deve ser realizada nas pacientes com valvulopatia, para prevenção da endocardite. Drogas vasoconstritoras ou preparados da ergotamina devem ser evitados ${ }^{1,3}$.

O período periparto é o mais crítico, com aumento do risco de morte materna devido a ruptura de aneurismas, insuficiência cardíaca congestiva, acidente vascular cerebral e insuficiência renal. A taxa de mortalidade materna pode chegar a $4,8 \%{ }^{1,2}$.

No puerpério, os cuidados devem ser voltados para os sinais de insuficiência cardiaca ou infecção. A amamentação é permitida, desde que a paciente não apresente complicações graves ${ }^{1.3}$.

A fertilidade não é afetada pela doença. O método de planejamento familiar adequado deve ser analisado individualmente, avaliando-se os aspectos obstétricos e cardiológicos ${ }^{1,6}$. 


\section{Referências}

\section{SUMMARY}

Takayasu's arteritis is an idiopathic occlusive inflammation of the aorta and its major branches. The disease shows a striking predilection for young women and thus is occasionally associated with pregnancy. The authors describe a case of a pregnant patient with Takayasu's arteritis. The pregnancy was accompanied by a multidisciplinary group in a satisfactory way. There was only one hospitalization due to an exacerbation of the symptoms during the $32^{\text {nd }}$ week of gestation, controlled by medical treatment. A vaginal delivery occurred at 37 weeks. A live infant weighing 2,750 $\mathrm{g}$ was delivered and the patient had an uncomplicated course.

KEY WORDS: Takayasu's arteritis. Pregnancy complications. Autoimmune diseases.
1. Bassa A, Desai DK, Moodley J. Takayasu's disease and pregnancy. S Afr Med J 1995; 85:107-12.

2. Ishikawa K, Matsuura S. Occlusive thromboartropathy (Takayasu's disease) and pregnancy: Clinical course and management of 33 pregnancies and deliveries. Am J Cardiol 1982; 50:1293-300.

3. De La Luna y, Olsen E, Pichardo Villilán G, Garcia Alonso A, Niz Ramos J. Sindrome de Takayasu y embarazo. Rev Med IMSS 1982; 20:387-93.

4. Wong VC, Wang RY, Tse TF. Pregnancy and Takayasu's arteritis. Am J Med 1983; 75:597-601.

5. Fignon A, Marret H, Alle C, Jacquet A, Avigdor S, Descamps $\mathrm{P}$, et al. A propos de l'association artérite de Takayasu, grossesse et maladie de Still. J Gynecol Obstet Biol Reprod (Paris) 1995; 24:747-50.

6. Ramana TS, Grupta U, Chalan J, Turndorf H. Anesthetic considerations in Takayasu's arteritis. Anesth Analg 1979; 58:247-9.

7. Costa AG, Magalhães CEF, Medina EAP, Barreto JLPB, Heuseler RAM. Doença de Takayasu e gestação: relato de caso. J Bras Ginecol 1997; 107:31-3. 